\title{
ENFERMEDADES INDUCIDAS POR DROGAS: ANEMIA APLÁSICA ADQUIRIDA, HEPATITIS MEDICAMENTOSA Y GASTRITIS HEMORRÁGICA EN EL HOSPITAL NACIONAL GUILLERMO ALMENARA IRIGOYEN AGOSTO I998 -OCTUBRE 200I
}

\author{
MIGUEL A. VALENCIA S., JUAN C. QUiSPE I., JESÚS V. RUMICHE B. \\ DEPARTAMENTO DE MICROBIOLOGIA Y PARASITOLOGIA BASICA Y APLICADA DE LA FACULTAD DE FARMACIA Y \\ BIOQUIMICA DE LA UNIVERSIDAD NACIONAL MAYOR DE SAN MARCOS.
}

\begin{abstract}
RESUMEN
El objetivo de la presente investigación fue establecer la relación causa - efecto entre la administración de fármacos y la aparición de Anemia Aplásica Adquirida, Hepatitis Medicamentosa y Gastritis Hemorrágica, identificando los fármacos probablemente causantes. Se aplicó el análisis deductivo mediante el Algoritmo de Karch y Lasagna modificado por Naranjo y colaboradores. Los criterins considerados para establecer relación de causalidad entre agente etiológico (medicamento) y la enfermedad inducida fueron: Relación temporal, entre el medicamento sospechoso y la enfermedad inducida por drogas; la resolución favorable de los signos, síntomas y pruebas de laboratorio después del retiro del agente causante probable; los datos de laboratorio y exámenes clínicos que relacionen, en el tiempo, a la droga y la aparición de las enfermedades en estudio. Se identificaron 18 casos de anemia aplásica adquirida ; 15 casos de hepatitis medicamentosa y 41 casos de gastritis hemorrágica. Se concluyó que el $27.8 \%$ de los casos de anemia aplásica y el $48.8 \%$ de los casos de gastritis hemorrágica tenían como agente etiológico probable a drogas. Los agentes quimioterápicos, con un $11.1 \%$ (5fluorouracilo, doxnrubicina, metotrexate) en anemia aplásica; los agentes antituberculosos con un $20.1 \%$ (isoniazida, rifampicina, pirazinamida, etionamida) en hepatitis medicamentosa y los AINE con un $29.3 \%$ (ibuprofeno, piroxican) en Gastritis hemorrágica, fueron los principales agentes probables causantes de las enfermedades estudiadas.
\end{abstract}

Palabras clave: anemia aplásica, gastritis hemorrágica, hepatitis medicamentosa.

\section{SUMMARY}

The goal of the present research was to establish the relationship cause - effect between drug administration and the presence of acquired aplasic anemia, drug hepatitis and hemorrhagic gastritis, identifying the probable causing drugs. It is applicd the deductive analysis by Karch and Lasagna algorithm modifyed by Naranjo et al. The considered criteria for establishing relationship of causality between ethiologic (drug) agent and induced disease were: temporal relationship between suspect drug and induced disease drugs; favorable resolution of the signs, symptoms and lab assay after retirement of probably causing agent; the lab data and clinical analysis that relate in time, the drug and the appearance of the diseases in the study. It was identified 18 cases of acquires aplasic anemia; 15 cases of drug hepatitis and 41 cases of hemorrhagic gastritis. It was concluded that $27,8 \%$ of the cases of aplasic anemia and $48,8 \%$ of the cases of hemorrhagic gastritis had as a probably ethiologic agent just drugs. The chemiotherapic agents with a 11,1\% (5- fluoracil, doxorubicina, metotrexate) in aplasic anemia; and antituberculosic agents with a 20,1\% (Isoniazide, Riphampicine , Pirazinamide, Etionamide) in drug hepatitis and the non steroidal antiimflamatory agents with a $29,3 \%$ (ibuprophen, Pyroxica) in hemorrhagic gastritis, were the probably main agents that causes the studied diseases from above.

Key words: aplasic anemia, hemorrhagic gastritis, drug hepatitis.

\section{INTRODUCCIÓN}

Esta investigación está enmarcada dentro de la Farmacoepidemiología, de la cual los estudios de utilización de medicamentos y la Farmacovigilancia son áreas importantes. Existen ciertas enfermedades causadas como consecuencia de reacciones adversas a fármacos o medicamentos, lo cual es un potencial problema en la salud de la población que las consume y cuando los médicos prescriben medicamentos; en esta situación, los principios epidemiológicos, en conjunción con el juicio clínico, pueden ser de ayuda.
Las compañías farmacéuticas se esfuerzan por identificar el efecto adverso antes de su comercialización, pero estos estudios se realizan en un número limitado de sujetos seleccionados -una muestra- sin patologías concomitantes, a dosis y condiciones controladas lo que hace que el espectro completo de efectos adversos sea desconocido. Estos efectos adversos más serios, inducidos por drogas, se presentan durante la etapa de comercialización, posterior a la obtención del Registro Sanitario, lo cual hacen necesarios los estudios epidemiológicos post comercialización, estudios de utilización de 
medicamentos (EUM), y la Farmacovigilancia, como actividad continua, aún cuando esto no resolverá el problema (1).

Se seleccionaron tres patologías cuya aparición está ligada al consumo de medicamentos de uso frecuente y hasta cierto punto indiscriminado de parte de los médicos, que las prescriben, y de los farmacéuticos, que las recomiendan, talvez por desconocimiento 0 por que no son conscientes de la magnitud del problema.

En 1888, se describió el primer caso de anemia aplásica por Erlich, quien refiere a una mujer de raza blanca de 21 años de edad, que presenta amenorrea, hemorragia retiniana, leucopenia y palidez intensa, que murió poco después de comenzar su enfermedad (2). losteriormente se publicaron casos similares de esta enfermedad con cuadros clínicos idénticos (leucopenia, trombocitopenia y anemia intensa), que ocurría como consecuencia de la exposición a productos tóxicos como benzol (Santenson, 1987). La anemia aplásica verdadera es un cuadro infrecuente, aproximadamente el $50 \%$ de los casos son ideopáticos y ocurren con mayor frecuencia en adultos y adolescentes jóvenes (3). En el $50 \%$ restante, la causa puede ser un agente químico (benceno, arsénico inorgánico), radiaciones o fármacos (3). Los fármacos implicados son antibióticos, antinflamatorios, anticonvulsivantes destacando el cloranfenicol, cuyo efecto adverso se manifiesta en la medula ósea (aplasia medular) y su incidencia es de 1 caso en 30 mil o más ciclos de tratamiento; el cuadro tóxico al parecer surge en individuos que reciben el fármaco por largo tiempo y sobre todo, los que estén expuestos a él en varias ocasiones(4). Otros fármacos que pueden ser causa de anemia aplásica son los antineoplasicos e inmunosupresores (antagonistas del ácido fólico, los agentes alquilantes, las antraciclinas y las nitrosureas, así como análogos de purina y pirinamina).

En cuanto a la hepatitis medicamentosa es un proceso inflamatorio y necrótico de las células hepáticas que puede ser producido por toxinas, virus, gran diversidad de medicamentos y agentes químicos. Es cada vez más frecuente el hallazgo de daño hepático inducido por medicamentos con presentación de una amplia gama de manifestaciones clínicas y bioquímicas, que van desde la simple elevación asintomática de las cifras de aminotransferasas hasta la presentación clínica de enfermedades como hepatitis aguda y necrosis hepática fulminante. Entre los múltiples efectos adversos de los medicamentos tenemos a la toxicosis hepátobiliar que constituye el $5 \%$ de todas las reacciones registradas por fármacos. Los patroncs clínicos y bioquímicos de la lesión pueden dar impresión de cualquier enfermedad hepática aguda o crónica (5).

Refiriéndonos a la gastritis hemorrágica, ésta se caracteriza por lesiones hemorrágicas a lo largo de la mucosa gástrica, debido a erosiones (6) . En general, son consecuencia de la ingesta de fármacos agresivos para la mucosa gástrica o de la agresión producida por alimentos, alcohol, infecciones o intoxicaciones. Los medicamentos más agresivos para la mucosa gástrica son los de mayor consumo habitual, como son los antiinflamatorios y el ácido acetilsalicílico (aspirina). Entre los primeros se distinguen dos clases: los no esteroideos y los corticoides. Su consumo, por vía oral o a través de inyecciones, suele ser agresivo para el estómago, ocasionando gastritis agudas, úlceras y hemorragias (4).

\section{MATERIAL Y MÉTODOS}

Es un estudio epidemiológico de utilización de medicamentos, transversal, retrospectivo, realizado en el Hospital Nacional Guillermo Almenara Irigoyen (H.N.G.A.I.) de ESSALUD.

Los materiales que se utilizaron fueron:

- Historias Clínicas de pacientes con diagnóstico de anemia aplásica, hepatitis medicamentosa y gastritis hemorrágica.

- Formatos para la recolección de la información diseñados específicamente para cada enfermedad en estudio.

- Algoritmo de Karch y Lasagna modificado por Naranjo y colaboradores.

Muestra: El universo lo constituía las historias clínicas de los pacientes hospitalizados en los diferentes servicios del HNGAI y diagnosticados con alguna de las tres patologías objetivos del estudio:

- Anemia Aplásica.- De agosto de 1998 - agosto del $2001-40$ casos.

- Hepatitis medicamentosa.- De enero de 1999 octubre del 2001 - 76 casos.

- Gastritis Aguda Hemorrágica.- De enero 2000 diciembre 2000 - 204 casos.

Criterios de inclusión: Historias clínicas de pacientes de ambos sexos con el diagnóstico de anemia aplásica, hepatitis medicamentosa y gastritis hemorrágica.

Criterios de exclusión: Historias clínicas de pacientes con datos incompletos o con otras patologías.

Método: Análisis de causalidad aplicando el algoritmo, el cual contempla:

- Secuencia temporal entre el (los) fármaco(s) sospechosos y la aparición del cuadro clínico.

- La plausibilidad de la relación de causalidad, teniendo en cuenta la descripción previa de la reacción en la literatura médica o las propiedades farmacológicas conocidas del medicamento. 
- El desenlace de la reacción después de la retirada del fámaco.

- La eventual repetición del episodio clínico con la readministración o reexposición al medicamento sospechoso y la posibilidad de que la reacción sea una manifestación de la patología de base del paciente o se relaciona con la prescripción del fármaco.

- Posibilidad de información adicional con exploraciones complementarias dirigidas a descartar otras etiologías de origen no farmacológicas.

Para confirmar que la historia dínica presenta un caso con la enfermedad en estudio se consideró los siguientes criterios para cada enfermedad:

\section{Anemia aplásica adquirida}

1. El diagnóstico clínico de anemia aplásica.

2. Confirmación del diagnóstico clínico con resultados de laboratorio (aspirado de médula ósea, dosaje de hemoglobina, hematocrito, entre otros) que se relacionen en un determinado periodo de tiempo con la administración del fármaco probable.

\section{Hepatitis medicamentosa}

1. Diagnóstico clínico de hepatopatía tóxica en la historia clínica y la descripción de los síntomas de hepatitis (náuseas, vómitos, ictericia).

2. Confirmación del diagnóstico clínico mediante pruebas bioquímicas como niveles elevados de aminotransferasas, bilitrubina y fosfatasa alcalina.

\section{Gastritis hemorrágica.}

1. El diagnóstico clínico de gastritis hemorrágica.

2. Confirmación del diagnóstico clínico, con resultados de laboratorio (endoscopia, hemoglobina entre otros) y que relacionen en determinado periodo de tiempo la enfermedad con la administración. del fármaco probable.

TABLA No1 - - Distribución por año de los casos de Anemia aplásica, Hepatitis medicamentosa y Gastritis hemorrágica, en el H.N.G.A.I., de agosto 1998 - octubre 2001

\begin{tabular}{|c|c|c|c|c|c|c|}
\hline \multirow{2}{*}{ ANOS } & \multicolumn{2}{|c|}{$\begin{array}{c}\text { ANEMIA } \\
\text { APLASICA }^{*}\end{array}$} & \multicolumn{2}{c|}{ HEPATITIS } & \multicolumn{2}{c|}{$\begin{array}{c}\text { GASTRITIS } \\
\text { MEDICAMENTOSA }\end{array}$} \\
\cline { 2 - 7 } & CASOS & $\%$ & CASOS & $\%$ & CASOS & $\%$ \\
\hline 1998 & 3 & 16.6 & 0 & 0 & 0 & 0 \\
1999 & 9 & 50 & 6 & 40 & 0 & 0 \\
2000 & 4 & 22.2 & 6 & 40 & 0 & 0 \\
2001 & 2 & 11.1 & 3 & 20 & 41 & 100 \\
TOTAL & 18 & $100 \%$ & 15 & $100 \%$ & 41 & $100 \%$ \\
\hline
\end{tabular}

\footnotetext{
Agosto 1998 - Agosto 2001

Enero 1999 - Octubre 2001

c. Enero 2000 - Diciembre 2000
}

\section{RESULTADOS}

Del total de 74 historias clínicas, revisadas en tres años de estudio, 18 casos correspondian a anemia aplásica, es importante mencionar que la anemia aplásica tiene una incidencia de 2 a 6 casos por millón de habitantes por año (6), siendo considerada una enfermedad de aparición rara ;15 casos correspondían a hepatitis medicamentosa, que se presentaron en 2 años y 10 meses. Por la falta de criterios exclusivos para el diagnóstico de hepatitis medicamentosa (7), es dificil que sea reportada como tal. Se identificaron 41 casos de gastritis hemorrágica (Tabla 1). 
Tabla No 2.- Distribución por sexo de casos en el H.N.G.A.I Agosto 1998 - Diciembre 2001

\begin{tabular}{|l|c|c|c|c|c|c|c|c|}
\hline \multirow{2}{*}{ SEXO } & Anemia Aplásica & \multicolumn{2}{c|}{$\begin{array}{c}\text { Hepatitis } \\
\text { medicamentosa }\end{array}$} & \multicolumn{2}{|c|}{$\begin{array}{c}\text { Gastritis } \\
\text { Hemorrágica }\end{array}$} & \multicolumn{2}{c|}{ TOTAL } \\
\hline Masculino & $\mathrm{N}^{\circ}$ & $\%$ & $\mathrm{~N}^{\circ}$ & $\%$ & $\mathrm{~N}^{\circ}$ & $\%$ & $\mathrm{~N}^{\circ}$ & $\%$ \\
Femenino & 9 & 50 & 8 & 53,3 & 21 & 51,2 & 38 & 51,35 \\
Total & 9 & 50 & 7 & 46,7 & 20 & 48,8 & 36 & 48,65 \\
& 18 & 100 & 15 & 100 & 41 & 100 & 74 & 100 \\
\hline
\end{tabular}

"Agosto 1998 - Agosto 2001; ' Enero 1999. Octubre 2001; 'Enero 2000 - Diciembre 2000

TABLA N*3.-Distribución por grupos de edad (años) en el H.N.G.A.I Agosto 1998 - Diciembre 2001

\begin{tabular}{|l|c|c|c|c|c|c|c|c|}
\hline $\begin{array}{c}\text { Grupo de Edad } \\
\text { (Años) }\end{array}$ & Anemia Aplásica & \multicolumn{2}{|c|}{$\begin{array}{c}\text { Hepatitis } \\
\text { medicamentosa }\end{array}$} & \multicolumn{2}{c|}{$\begin{array}{c}\text { Gastritis } \\
\text { Hemorrágica }\end{array}$} & \multicolumn{2}{c|}{ TOTAL } \\
\hline & $\mathrm{N}^{\circ}$ & $\%$ & $\mathrm{~N}^{\circ}$ & $\%$ & $\mathrm{~N}^{\circ}$ & $\%$ & $\mathrm{~N}^{\circ}$ & $\%$ \\
0 a 10 & - & & 1 & 6,7 & - & 1 & 1,35 \\
11 a 20 & 8 & 44,5 & 1 & 6,7 & 1 & 2,5 & 10 & 13,5 \\
21 a 40 & 4 & 22,25 & 2 & 13,3 & 1 & 2,5 & 7 & 9,45 \\
41 a 60 & 4 & 22,25 & 8 & 53,3 & 17 & 41,5 & 29 & 39,2 \\
61 a más & 2 & 11 & 3 & 20 & 22 & 53,5 & 27 & 36,5 \\
Total & 18 & 100 & 15 & 100 & 41 & 100 & 74 & 100 \\
\hline
\end{tabular}

"Agosto 1998 - Agosto 2001; $\quad$ "Enero 1999 - Octubre 2001; $\quad$ "Enero 2000 - Diciembre 2000

Tabla $N^{\circ}$ 4.- Agentes probables causantes de Anemia aplásica adquirida, Hepatitis medicamentosa y gastritis hemorrágica en el H.N.G.A.I Ágosto 1998 - Diciembre 2001

\begin{tabular}{|l|l|l|l|l|l|l|}
\hline $\begin{array}{l}\text { Agente } \\
\text { probable }\end{array}$ & \multicolumn{2}{|c|}{ Anemia aplásica } & \multicolumn{2}{|c|}{$\begin{array}{c}\text { Hepatitis } \\
\text { medicamentosa }\end{array}$} & \multicolumn{2}{|c|}{$\begin{array}{c}\text { Gastritis } \\
\text { hemorrágica }\end{array}$} \\
\hline & $\mathrm{N}^{\circ}$ & $\%$ & $\mathrm{~N}^{\circ}$ & $\%$ & $\mathrm{~N}^{\circ}$ & $\%$ \\
E. por medic. & 5 & 27.8 & 14 & 93.3 & 20 & 48.8 \\
Medic totros & 0 & 0 & 1 & 6.7 & 7 & 17.1 \\
E.no I. Medic & 5 & 27.8 & 0 & 0 & 12 & 29.3 \\
No identificado & 8 & 44.4 & 0 & 0 & 2 & 4.8 \\
TOTAL & 18 & 100 & 15 & 100 & 41 & 100 \\
\hline
\end{tabular}

* Enlermedades inducidas por medicamento

'Enfermedades no inducidas por medicamentos

Tabla $\mathrm{N}^{\circ}$ 5.- Intervalo de tiempo entre la exposición al agente probable (medicamento) y la aparición de la enfermedad Agosto 1998 - Diciembre 2001

\begin{tabular}{|c|c|c|c|c|c|c|}
\hline \multirow[t]{2}{*}{$\begin{array}{l}\text { Intervalo } \\
\text { (semanas) }\end{array}$} & \multicolumn{2}{|c|}{ Anemia aplásica } & \multicolumn{2}{|c|}{$\begin{array}{c}\text { Hepatitis } \\
\text { medicamentosa }\end{array}$} & \multicolumn{2}{|c|}{$\begin{array}{l}\text { Gastritis } \\
\text { hemorragica }\end{array}$} \\
\hline & $\mathrm{N}^{\circ}$ & $\%$ & $\mathrm{~N}^{\circ}$ & $\%$ & $\mathrm{~N}^{\circ}$ & $\%$ \\
\hline <a 1 sem. & - & - & 0 & & 16 & 59.3 \\
\hline $\begin{array}{l}1 \text { sem. } \\
22.2\end{array}$ & & - & & 4 & 26.5 & 6 \\
\hline 2 sem. & - & - & 4 & 26.5 & 1 & 3.7 \\
\hline 2 a $<4$ sem. & 3 & 60 & 2 & 13.5 & 1 & 3.7 \\
\hline 4 a 8 sem. & 1 & 20 & 4 & 26.5 & 0 & \\
\hline $10-12 \mathrm{sem}$. & 1 & 20 & 1 & 7 & 0 & \\
\hline $13 a+$ & - & - & - & & 0 & \\
\hline No especifica & - & - & - & & 3 & 11.1 \\
\hline TOTAL & 5 & 100 & 15 & 100 & 27 & 100 \\
\hline
\end{tabular}


Tabla $\mathrm{N}^{0}$ 6.- Evolución de la enfermedad en estudio en el H.N.G.A.I Agosto 1998 - Diciembre 2001

\begin{tabular}{|l|c|l|l|l|l|l|}
\hline Evolución & \multicolumn{2}{|c|}{ Anemia aplásica } & \multicolumn{2}{|c|}{$\begin{array}{c}\text { Hepatitis } \\
\text { medicamentosa }\end{array}$} & \multicolumn{2}{|c|}{$\begin{array}{c}\text { Gastritis } \\
\text { hemorrágica }\end{array}$} \\
\hline & $\mathrm{N}^{\circ}$ & $\%$ & $\mathrm{~N}^{\circ}$ & $\%$ & $\mathrm{~N}^{\circ}$ & $\%$ \\
Estacionario & 11 & 61.1 & 2 & 13.3 & 24 & 58.5 \\
Favorable & 6 & 33.3 & 11 & 73.4 & 16 & 39 \\
Fallece & 1 & 5.6 & 2 & 13.3 & 1 & 2.5 \\
TOTAL & 18 & 100 & 15 & 100 & 41 & 100 \\
\hline
\end{tabular}

Tabla 7.- Agente ¿Causas probables de anemia? aplásica, hepatitis medicamentosa y gastritis hemorrágica. En el H.N.G.A.I. Agosto 1998 - Diciembre 2001

\begin{tabular}{|c|c|c|c|c|c|c|c|}
\hline \multicolumn{2}{|c|}{ HEP'ATITIS MEDICAMENTOSA } & \multicolumn{3}{|c|}{ GASTRITIS HEMORRÁGICA } & \multicolumn{3}{|c|}{ ANEMIA APLÁSICA } \\
\hline Agente causal & $\%$ & Agente causal & $\mathrm{N}^{\mathbf{0}}$ & $\%$ & Agente causal & $N^{o}$ & $\%$ \\
\hline Ranitidina & 6.7 & AINE no diferenciado & 3 & 7.3 & Valproato de sodio & 1 & 5.6 \\
\hline Paracetamol & 13.3 & Paracetamol+AINE & 2 & 4.9 & + Fenitoina & & \\
\hline Clorpromazina & 6.7 & Ciprofloxacino & 1 & 2.4 & Transplante renal & 1 & 5.6 \\
\hline Fenitoina & 13.3 & Ibuprofeno+Piroxicam & 1 & 2.4 & Insecticidas agricolas & 1 & 5.6 \\
\hline $\mathrm{INH}+\mathrm{RFM}+\mathrm{PZD}$ & 6.7 & Bbuprofeno & 5 & 12.2 & Agentes quimioterápiocos & 2 & 11.1 \\
\hline PZD+RFM & 6.7 & Piroxicam & 3 & 7.3 & Cloranfenicol & 1 & 5.6 \\
\hline Etionamida & 6.7 & Warfarina & 1 & 2.4 & Embarazo & 1 & 5.6 \\
\hline Metimazol & 6.7 & Aspirina & 4 & 9.8 & Cotrimoxazol & 1 & 5.6 \\
\hline Ketoconazol & 13.3 & Alcohol+AINE & 1 & 2.4 & Hepatitis & 1 & 5.6 \\
\hline Cotrimazol & 6.7 & Ibuprofeno+alcohol & 1 & 2.4 & Virus Epstein Barr & 1 & 5.6 \\
\hline Amitriptilina/alcohol & 6.7 & $\mathrm{AAS}+\mathrm{HP}$ & 1 & 2.4 & 10.- No identificado & 8 & 44.4 \\
\hline Captopril & 6.7 & Antibiótico $+\mathrm{HP}$ & 1 & 2.4 & TOTAL & 18 & 100 \\
\hline TOTAL & 100 & Ibuprofeno+HP & 1 & 2.4 & & & \\
\hline & & Piroxican+HP & 1 & 2.4 & & & \\
\hline & & Alcohol $+\mathrm{HP}$ & 1 & 2.4 & & & \\
\hline & & Cotrimoxazol+HP & 1 & 2.4 & & & \\
\hline & & Alcohol & 7 & 17.1 & & & \\
\hline & & Helicobacter piloni $(\mathrm{HP})$ & 2 & 4.9 & & & \\
\hline & & IRCT & 1 & 2.4 & & & \\
\hline & & Cirrosis cardiaca & 1 & 2.4 & & & \\
\hline & & No identificado & 2 & 4.9 & & & \\
\hline & & TOTAL & 41 & 100 & & & \\
\hline
\end{tabular}

\section{Discusión}

La incidencia y presentación de casos puede variar notablemente por varios factores como la diferencia poblacional, las diferentes épocas en que se realiza el estudio, la forma en que son reportados los casos o incluso los criterios de inclusión considerados. No fue posible determinar la incidencia porque para calcularla se necesita conocer la cantidad total de población expuesta, dato con el cual no se contaba.

En anemia aplásica se encontró una distribución equilibrada por sexo, $50 \%$ para varones y mujeres respectivamente (Tabla 2); a diferencia de otro estudio desarrollado en el medio (8) donde el sexo femenino era el más afectado. En un estudio realizado en Europa Occidental e Israel, el grupo de 25 años fue el más afectado, no concordando con lo obtenido en esta investigación, donde el grupo de edad entre 10 20 años $(44,4 \%)$ adquirió esta patología (Tabla 3). Asimismo, en el $44 \%$ de los casos (Tabla 4) no se identifica la causa durante el interrogatorio al paciente, denominándoseles casos de anemia aplásica idiopática (9). En Europa y Estados Unidos del $40 \%$ al $70 \%$ de los casos de anemia aplásica son. idiopáticos; en Japón más del $90 \%$ de los casos son de esta naturaleza (10). 
El efecto adverso más temido del cloranfenicol es la anemia aplásica adquirida (11). Se reporta un caso de anemia aplásica relacionada con cloranfenicol, que representa el $5.6 \%$ de los casos (Tabla 7), la enfermedad apareció a los tres meses de haberse iniciado la terapia con cloranfenicol, por lo que podría tratarse de una anemia aplásica tardía inducida por cloranfenicol. En un estudio sobre tratamiento de fiebre tifoidea con cloranfenicol (droga de elección) en una serie de 598 pacientes, el $6 \%$ de los casos presentó alteraciones hematológicas durante el tratamiento que obligó a cambiar o a reducir la dosis de la droga (12). La bibliografía refiere que la anemia aplásica puede presentarse en 1:40,000 y 1:25,000 tratamientos con cloranfenicol (13). Se presentó un caso relacionado con anticonvulsivantes (valproato de sodio y fenitoina) que habian sido utilizados para el control de la convulsión y los medicamentos habían sido administrados dentro de los tres meses previos a la aparición de los primeros síntomas de la anemia aplásica. No se pudo diferenciar que droga específicamente, causó la anemia aplásica, pués las dos drogas fueron administradas simultáneamente, y ambas drogas tienen antecedentes de haber inducido a anemia aplásica, pero sólo en casos aislados $(14,1 \bar{j})$. Se encontró un caso relacionado con Cotrimoxazol, fármaco que puede inducir anemia aplásica $(5,14,16,17,18)$. Encontramos que 5 casos $(27,8 \%)$ estaban relacionados con fármacos (Tabla 4), de los cuales, en 3 casos la enfermedad aparece entre la segunda y cuarta semana después de haberse administrado la droga (Tabla 5) y un paciente fallecido a causa de la anemia aplásica (Tabla 6), el cual tenía una relación causal con hepatitis; la bibliografía menciona que el $80 \%$ de los casos de anemia aplásica relacionados con hepatitis son fatales (9).

En este estudio, el $53.3 \%$ de los casos cran varones, mientras el $46.7 \%$ eran mujeres (Tabla 2); locual puede deberse a varios factores, como la diferente forma de reportar la enfermedad hepática inducida por drogas pues no hay aún criterios definidos para su diagnóstico.

Con respecto a la edad, en un estudio realizado en el país, de hepatotoxicidad inducida por drogas antituberculosas, se refiere que hay predominio en el grupo etáreo joven y de sexo masculino(19); en este estudio, se determinó que el grupo de edad que predominó fue el de entre 41 y 60 años (Tabla 3 ).

El paracetamol está extensamente disponible y es una de las drogas usualmente más usadas en el tratamiento de la fiebre. El acceso fácil y la familiaridad general con el paracetamol contribuyen a la incidencia alta de dosis excesiva en la población. En un estudio realizado en dos hospitales pediátricos, en 10 años de estudio, se encontraron 322 casos reportados como hepatotoxicidad asuciada con paracetamol (20), de los cuales el $35.5 \%$ eran varones y el $64.5 \%$ eran mujeres. En el presente estudio, en casi tres años de estudio, se halló dos casos de hepatotoxicidad asociada con paracetamol que representa el $13.3 \%$ de los casos de hepatotoxicidad inducida por drogas (Tabla 7). Se consideramos que son pocos casos de hepatotoxicidad asociada con paracetamol en relación con el trabajo anteriormente citado, esto se puede deber al tipo de diseño de investigación empleado que en ese caso era prospectivo y en el presente, retrospectivo; al tipo de criterios definidos para el diagnóstico de hepatitis medicamentosa o a que el paciente tuvo una evolución favorable a corto tiempo y no se tomó en cuenta la reacción adversa (21).

Otros fármacos relacionados con esta enfermedad son las drogas antituberculosas, lo cual se evidenció en un estudio realizado en el Hospital Cayetano Heredia (19) donde se reportó que el $71.8 \%$ de los casos estuvieron relacionados con drogas antituberculosas, a diferencia de lo encontrado en esta investigación en que tres casos $(20,1 \%$ ) ( Tabla 7 ) de hepatitis inducida por drogas estaban relacionados con drogas antituberculosas. La isoniazida, rifampicina y pirazinamida son drogas que potencialmente pueden producir una reacción de hepatotoxicidad y si son administradas simultáneamente no será posible identificar con facilidad cual de estas drogas es el agente causal. Esto se presentó, encontrando un caso que se relaciona con la administración simultánea de estas tres drogas y otro caso que estaba relacionado con pirazinamida y rifampicina; estos dos casos representan el $13.4 \%$ del total de los casos (Tabla 7).

Por otro lado, se sabe que la Etionamida puede causar aumentos en las concentraciones de bilirrubina, AST y ALT en la sangre de los pacientes, que generalmente es reversible con la interrupción de la droga (22)y esto se verificó en este estudio identificándose un caso que se reportaba como hepatotoxicidad inducida por Etionamida. La bibliografía menciona que la prevalencia de la hepatitis inducida por Etionamida es del 3-5\% de los pacientes que la consumen y el aumento de las enzimas séricas ocurre en más del $30 \%$. Normalmente, la hepatitis se inicia después de varios meses de terapia(23).

El ketoconazol liene una incidencia de 1 en 10,000 tratamientos de inducir hepatotoxicidad, la duración media de la terapia con ketoconazol en pacientes que desarrollaron la hepatotoxicidad con presencia de síntomas era aproximadamente 28 días. La lesión hepática normalmente ha sido reversible después de la interrupción del tratamiento del ketoconazol. Se han informado varios casos de hepatitis en niños (24). 
En el presente estudio se ha encontrado dos casos que se relacionan con ketoconazol que representa el $13.3 \%$ de los casos de hepatitis medicamentosa.

La necrosis hepatocelular inducida por fenitoina es rara, pero a menudo fatal. Esta normalmente acompañada por otros signos de hipersensibilidad, frecuentemente como parte de un síndrome difuso de linfadenopatia, de dermatitis exfoliante, fiebre y leucocitosis. Normalmente, el inicio se desarrolla en las 8 primeras semanas de terapia (24). En este estudio se identificaron dos casos de hepatotoxicidad inducida por fenitoina, lo que representa el $13.3 \%$ de los casos (Tabla 7), similares a los que la bibliografía reporta; es necesario mencionar que un signo que se presentó, en los dos casos, fue la dermatitis que caracteriza a la hepatotoxicidad inducida por fenitoina, los dos casos tuvieron una evolución favorable tras la suspensión de la droga.

El captopril es una droga que puede producir daño hepatobiliar, produciendo ictericia, hepatitis además de raros casos de necrosis y colestasis(24). El caso encontrado de hepatitis medicamentosa relacionado con captopril (Tabla 7), era una paciente con diagnóstico de: hipertensión, iniciando su terapia antihipertensiva con captopril y su evolución fue estacionaria. (Tabla 6).

Un estudio en 12 voluntarios sanos da referencia que, en 6 de los casos, la ranitidina, aumentó, en dos veces, los valores de ALT (alanino amino transferasa) con respecto a los valores antes de recibir el tratamiento, con dosis de $100 \mathrm{mg} /$ día / siete días. Existen informes ocasionales de colestasis, o las hepatitis mixtas, con o sin la ictericia. En tales circunstancias, debe descontinuarse la ranitidina inmediatamente. Estos eventos son normalmente reversibles (24). El caso reportado de hepatitis medicamentosa inducida por ranitidina representa el $6.7 \%$ de los casos (Tabla 10).

La amitriptilina, es una droga que muy raramente induce una hepatitis medicamentosa (24), pero ésta puede interactuar con el alcohol produciendo una disminución de su metabolismo. En el presente estudio se encontró un caso de hepatotoxicidad relacionada con amitriptilina-alcohol, que representa el $6.7 \%$ de los casos, la bibliografía no da referencia exacta a cerca de si ésta interacción puede incrementar el riesgo de causar hepatotoxicidad.

El metimazol puede inducir una hepatitis con ictericia que puede ser persistente por varias semanas después de suspendida la droga (24); en nuestro estudio el caso de hepatitis medicamentosa que estaba relacionado con metimazol (Tabla 7) presentó ictericia pero su evolución fue favorable tras la suspensión de la droga.
El Cotrimoxazol, ampliamente utilizado por nuestras poblaciones, es otra de las drogas que puede inducir hepatotoxicidad, incluso colestasis, ictericia, hasta necrosis hepática, lo que se evidencia con elevación de transaminasas y bilirrubina en suero $(14,24)$, tal como sucedió en el caso de hepatitis medicamentosa inducido por cotrimoxazol (Tabla7).

La Clorpromazina puede producir una ictericia leve ocasionalmente con prurito, y suele aparecer después de la segunda o cuarta semana de haberse administrado la droga, al parecer es una reacción de hipersensibilidad y no hay correlación con la dosis (11). La hepatotoxicidad por clorpromazina es una reacción colestásica idiosincrásica. Alrededor del 1 $\%$ de los pacientes que reciben clorpromazina presentan colestasis intrahepática con ictericia; en raras ocasiones se ha detectado la aparición de ictericia tras la administración de una dosis (4).

En el caso de Metotrexate, aunque el período de latencia entre la exposición al fármaco y la presentación de la hepatotoxicidad varía de pocos días a años, debe considerarse como especialmente sospechosos a las drogas consumidas en los 3 meses previos. En el $66 \%$ de los casos de hepatitis medicamentosa presentados, el intervalo de tiempo entre la administración de la droga y la aparición de la hepatitis medicamentosa varía entre una y dos semanas, y el 7\% $(n=1)$ de los casos fue entre 10 y 12 semanas (Tabla 5).

La evolución final en el $73.3 \%$ fue favorable, mientras que un $13.3 \%$ de los pacientes tuvo una evolución estacionaria (Tabla 6); es necesario mencionar que los fármacos pueden inducir una hepatopatía inducida por drogas que se puede hacer crónica y el $13.3 \%(n=1)$ de los pacientes fallece con una hepatopatia crónica asociada con otra complicación.

La gastritis hemorrágica es la causa del 23\% de los casos de hemorragia digestiva alta (25).Un estudio realizado en el Hospital Nacional Guillermo Almenara Irigoyen, durante los últimos años, con 1692 casos de hemorragia digestiva alta, arroja como resultado que el $64 \%$ de casos eran del sexo masculino y el $36 \%$ era del sexo femenino. Mientras el $50 \%$ estaba conformado por pacientes mayores de 60 años. Se comprobó que la principal causa de hemorragia digestiva alta era la ulcera gástrica (25).

Un estudio de hemorragia digestiva alta en niños da como resultado que el $52.6 \%$ se presenta en varones (26). En el presente estudio se encontró que un $51.2 \%$ eran varones mientras que el $48.8 \%$ eran mujeres, se puede observar que hay una diferencia pequeña entre el porcentaje de casos de mujeres con respecto al de varones (Tabla 1). 
Se observó que el mayor número de casos se presenta entre las edades de 70 - 80 años con $31.7 \%$ y que el $53.6 \%$ de los casos eran pacientes mayores de 60 años (Tabla 2), lo que concuerda con las referencias bibliográficas $(4,27,28)$ que mencionan que hay mayor riesgo de presentar gastritis hemorrágica en pacientes mayores de 60 años.

Un estudio realizado en el Instituto Nacional de Salud del Niño, reporta que el $36.4 \%$ de los casos de hemorragia digestiva alta estaba relacionado con el consumo de medicamentos(26). Otro estudio, realizado en pacientes con lesiones gástricas, reportó que un $50 \%$ de los casos estaba relacionado con el consumo de medicamentos, alcohol o ambos (29).

En el presente estudio, el $48,8 \%$ de los casos están relacionados con el consumo de sólo medicamentos, mientras que un $4,9 \%$ esta relacionado con el consumo concomitante de alcohol con medicamentos y un $12.2 \%$ de los casos de gastritis hemorrágica está relacionado con el consumo de medicamentos y Helicobacter pylori (Tabla 7).

Del análisis de 10 estudios caso-control $(8,9,30)$ se desprende que el riesgo relativo de padecer una complicación hemorrágica, entre los que ingieren AINE, oscila entre una amplia banda que va de 2,2 a 13,7 sí bien la mayoría se sitúa alrededor de 4 a 5 ; un problema de estos estudios es que la mayoría no realiza distinción entre el tipo de AINE consumido.

"Un estudio realizado sobre hemorragia digestiva en el Hospital Nacional Cayetano Heredia, da como resultado que la hemorragia digestiva inducida por AINE era de un 19.7\%, con un porcentaje similar asociado con el consumo de bebidas alcohólicas (31); otro estudio realizado sobre la hemorragia por ulcera duodenal o gástrica, de los pacientes incluidos en el estudio, el 30.6\% de los casos tenían antecedentes de ingestión de AINE»(32).

La infección por Helicobacter pylori $\left(\mathrm{H}^{*}\right)$ y la ingesta de Antiinflamatorios No Esteroideos (AINE) son, en la actualidad, los dos factores etiológicos más importantes en la cnfermedad ulcerosa péptica. La infección por $\mathrm{HP}$ es una de las más prevalentes en el mundo, afectando entre $20-80 \%$ de la población general, en función de factores ambientales, étnicos y socioeconómicos (32) sobre todo en los mayores de 50 años. Por ello es frecuente que los pacientes tratados con AINE presenten también infección por HP. En nuestro estudio encontramos que el $4.9 \%$ de los casos tenían como agente etiológico probable solo a Helicobacter pylori (Tabla N $\mathrm{N}^{\circ}$ ).

En diversos estudios sobre hemorragia digestiva, no diferencian a los AINE entre sí. $(31,32)$. En el presente estudio, el AINE relacionado con el mayor número de casos de gastritis hemorrágica, es el Ibuprofeno que representa el $12.2 \%$ de los casos. También indicamos que $2.4 \%$ de los casos está relacionado con el consumo concomitante de ibuprofeno con alcohol y otru $2.4 \%$ de los casos relacionados con ibuprofeno y la bacteria helicobacter pylori (Tabla No7). Asimismo encontramos que el $9.8 \%$ de los casos tenían como agente etiológico probable al ácido acetil salicílico (Aspirina), con dosis de $250 \mathrm{~g} \mathrm{c} / 24$ y otros $4.8 \%$ de los casos estaba relacionado con el consumo de AINE y Helicobacter pylori.

En un estudio con 2300 pacientes aproximadamente el $22.5 \%$ de los pacientes que recibieron dosis diarias de $20 \mathrm{mg}$ de piroxicam por más de un año tuvieron problemas gastrointestinales(26) a diferencia de lo hallado en nuestro estudio en que el $7.3 \%$ de los casos estaba relacionado con el consumo de piroxicam, siendo la segunda droga que estuvo relacionado con más casos de gastritis hemorrágica aguda, después del ibuprofeno. Además es precio mencionar que otros $2.4 \%$ de los casos estaba relacionado concomitantemente con el piroxicam y la presencia del helicobacter pylori .

La warfarina es un anticoagulante de estructura cumarínica, que ocasionalmente produce como efecto adverso hemorragias menores, señalado como del 2$9 \%$ de los efectos adversos de la warfarina. En el presente estudio se encontró que el $2.4 \%$ de los casos estaba relacionado con la warfarina como agente etiológico probable.

En un estudio realizado con 2799 pacientes que recibicron ciprofloxacino, el $7.9 \%$ de los pacientes tuvieron efectos adversos y el $1.5 \%$ de los efectos adversos estaban relacionados con problemas gastrointestinales, entre los que se puede mencionar el sangrado gástrico.(26) En el presente estudio el $2.4 \%$ de los casos estaba relacionado con ciprofloxacino como agente etiológico probable. El alcohol y el helicobacter pylori también son considerados como agentes etiológicos de causar sangrado gástrico, aunque aún no esta probado fehacientemente, como si es el caso de los AINE y otros medicamentos. La gastritis aguda erosiva puede ser inducida a parte de ser secundaria a la ingesta de medicamentos, alcohol o la presencia de Helicobacter pylori, también puede ser secundaria a cuadros patológicos graves con repercusión multiorgánica también denominados los casos de gastritis por estrés(4). En el presente estudio encontramos que el $4.9 \%(n=2)$ de los casos estaba relacionado con un cuadro patológico, como son los problemas de cirrosis cardiaca e insuficiencia renal crónica terminal .

Del análisis realizado se concluye que el $27.8 \%$ de los casos de anemia aplásica, el 93,3\% de Hepatitis medicamentosa y el $48.8 \%$ de los casos de gastritis hemorrágica estaban relacionados con el consumo de fármacos y los medicamentos probables causantes 
en un $11.1 \%$ de los casos de anemia aplásica fueron los agentes quimioterápicos (5-fluorouracilo, doxorubicina, metotrexate) ; los agentes antituberculosos con un $20.1 \%$ ( isoniazida, rifampicina, pirazinamida, etionamida) en hepatitis medicamentosa y los AINE con un $29.3 \%$ (ibuprofeno, piroxicam) en Gastritis hemorrágica.

\section{REFERENCIAS BIBLIOGRÁFICAS}

1. Kaufman, DW.; Shapiro, S. 2000. Epidemiological assessment of drug-induced disease. The Lancet. Volume 356(9238), 1339-1343.

2. Sans, J.; Rozman, C. 1995. Anemia Aplastica, Eritroblastopenias, Amegacariocitosis. Hematología Clínica. Tercera edición. Editorial Barcelona: pp. 251 - 261.

3. Bees, MH. ; Berkow ,R. 1999 El Manual MERCK. Décima Edición Española. Madrid. Harcourt.

4. Harrison, I.; Braunwald; Wilson;Martín; Fauci; Kosper. 1998. Principios de Medicina Interna. Décima edición en ingles. España. Mc GRAW HILL.

5. Yamada Tadataka. 2000 Manual de Gastroenterología. México DF. Mc Graw Hall Latinoamericana.

6. Hennessy, S ; Kinman, J L.; Berlin, J A.; Feldman, H.; Carson, J L.; Kimmel, S.; Farrar, J.; Harb, G.; Strom, B.1997. Lack of Hepatotoxic Effects of Parenteral Ketorolac in the Hospital Setting. American Medical Association. 57(21): pp. 25102514.

7. Tovar J L., Erill S., et al. 1998. Bases de terapéutica 4. Medecine; 18: 95-102.

8. Baldeón E. 1993. Anemia Aplasica Adquirida. Tesis para obtener el título de Médico Cirujano. Universidad San Martín de Porres. Lima.

9. Velez A., Rojas W., Borrero R., Restrepo J.1994. Anemia Aplastica - Insuficiencia de la Medula Osea - Pancitopenia, Fundamentos de Medicina (Hematología). Cuarta edición. Editorial Carvajal. pp. 65 - 73. Cordoba.

10. Camitta B. Storb R.1982. Aplastic anemia. N. Eng J. Med. 306: 712-714.

11. Hardman J G., Limbird L. E., Gilman A. G.1998. Goodman E Gilman's. Las Bases Farmacológicas de la terapéutica. Novena edición. México D.F. Mc GRAW - HILL, Interamericana.
12. Gotuzzo E., Guerra J. 1981. Tratamiento de la Fiebre Tifoidea. Diagnóstico 8(4): 195-201.

13. Jacobs, R. A. \& Guglielmo J. 2000. Anti-infective Chemotherapeutic $\mathcal{E}$ Antibiotic Agents. Current Medical Diagnosis \& Treatment 39th Edition. The McGraw-Hill Companies.

14. Montvale NJ. 2000. Physicians Desk Reference. 54 Th ed. Medical Economics Company.Inc. Canadá.

15. Ministerio de Salud /Dirección General de Medicamentos, Insumos y Drogas/ Sistema Peruano de Farmacovigilancia. 2000. Algoritmo de decisión para la evaluación de la relación de causalidad de una reacción adversa a medicamentos. Lima .

16. Montvale NJ. 1995. Sulfamethoxazole and trimethoprim (Sulfatrim-DS, Interstate). Red book 1995. Medical Economics Data. : 426-7. Canadá.

17. Product Information: Bactrim, trimethoprim and sulfamethoxazole. Roche Pharmaceuticals, Nutley, NJ, USA. Rev. 08/1998.

18. Mc Evoy G. A., Pharm D. 2000. Cotrimoxazol. AHFS Drug Information 2000. Editorial Staff. New York.

19. Espinoza F. 1983. Hepatotoxicidad por drogas antituberculosas, Hospital de Apoyo Cayetano Heredia.Tesis para obtener el Grado de Bachiller en Medicina. Universidad Particular Cayetano Heredia. Lima.

20. Alander, SW. MD.; Dowd, M. D MD, MPH; Bratton, SL. MD; Kearns, GL. PharmD.2000 Pediatric Acetaminophen Overdose: Risk factors associated with Hepatocellular injury. American Medical Association. 154(4): 346-350.

21. Chavez A. Hepatitis toxica por drogas. 1994 Hospital de Apoyo Cayetano Heredia. Tesis para obtener el Grado de Bachiller en Medicina. Universidad Peruana Cayetano Heredia. Lima.

22. Mc Evoy ,G. Ethionamide. 2001. AHFS Drug Information 2000. Editorial Staff . New York.

23. Mosby's Genrxä . 2000. The Complete reference for Generic And Brand Drugs - 10th Ed.

24. Andrade, RJ y Lucena, MI. 2001. ¿Es inevitable la hepatotoxicidad inducida por fármacos?. Revista Española De Enfermedades Digestivas. Vol. 93, N. ${ }^{\circ} 1: 48-50$.

25. Gestión Médica. 22-28 de octubre del 2001. Estudio revela aumento de casos de sangrado 
gástrico en el país. Gestión Médica. Edición 243. Año 6. Lima.

26. Reyes Alpaca.1995. Hemorragia Digestiva Alta en niños en el Instituto Nacional de Salud del Niño abril 1985 - marzo 1995. 'Tesis para obtener el Grado de Bachiller en Medicina. UPCH. Lima.

27. Olaeta E; López A; Moran S. 1995. Ulcera péptica y antiinflamatorios no esteroideos. Rev gastroenterol Mex. 60 (supl 3): 40.

28. Tierney, LM Jr., MD; McPhee, SJ. MD, Papadakis, MA, MD.2000.CURRENT MEDICAL DIAGNOSIS \& TREATMENT - 39th Ed. Copyright ã 2000 by The McGraw-Hill Companies. London.

29. Kohatsu D.1986. Aspectos clínicos, radiológicos, endoscópicos e histopatológicos de las lesiones elevadas gástricas diagnosticadas en el Hospital Arzobispo Loayza de 1980 a 1985 . Tesis para obtener el Grado de Bachiller en Medicina. UPCH. Lima.

30. Handbook of adverse drug interactions . 2000. The Medical I etter. Copyright(c) New York.

31. Mendez C.1996. Hemorragia digestiva en el Hospital Nacional Cayetano Heredia 1994-1995. Tesis para obtener el Grado de Bachiller en Medicina. UPCH. Lima.
32. Hoyos, A. ;Arango, H.; Rengifo, E. 1998 Proporción de resangrado y letalidad temprana después de inyección endoscópica en hemorragia por úlcera duodenal o gástrica. Colombia Médica. 29: 14-17.

33. Kohatsu D. Aspectos clínicos, radiológicos, endoscópicos e histopatológicos de las lesiones elevadas gástricas diagnosticadas en el Hospital Arzobispo Loayza de 1980 a 1985. 1986 Tesis para obtener el Grado de Bachiller en Medicina. UPCH. Lima .

34. HANDBOOK OF ADVERSE DRUG INTERACTIONS (2000). The Medical Letter. Copyright(c) 2000. New York

35. Mendez C. Hemorragia digestiva en el Hospital Nacional Cayetano Heredia 1994-1995. Tesis para obtener el Grado de Bachiller en Medicina. UPCH. Lima.

36. Hoyos, A. ;Arango, H.; ,Rengifo, E. 1998 Proporción de resangrado y letalidad temprana después de inyección endoscópica en hemorragia por úlcera duodenal o gástrica. Colombia Médica. 29: 14-17.

37. Sánchez D. Hepatitis Tóxica. Con acceso el 10 de enero del 2002. De www.fepafem.org/guias/ 5.13.html <http://www.fepafem.org/guias/ 5.13.html>. 